\title{
The Third Asian Radiology Forum: Screening Programs
} in Asia

\author{
Ning Chien, $M D^{1,2}$, Jo-Yu Chen, $M D^{1,2}$, Shyh-Jye Chen, $M D$, PhD ${ }^{1,2}$, Yeun-Chung Chang, $M D, P^{1,2}$ \\ ${ }^{1}$ Chinese Taipei Society of Radiology (CTSR), Taipei, Taiwan; ${ }^{2}$ Department of Medical Imaging, National Taiwan University Hospital, National \\ Taiwan University College of Medicine, Taipei, Taiwan
}

According to reports presented at the Asian Radiology Forum 2017 in Taipei, the landscape of screening in Asia is growing, and the role of radiologists is also evolving. The Asian Radiology Forum was first held by the Korean Society of Radiology and the national delegates of Asian radiological partner societies, who attended this meeting with the aim of discussing selected subjects of global relevance in radiology. In 2017, current trends and policies concerning various cancer screening programs from each delegate country were discussed. Various screening programs were discussed, including liver screening in Thailand, thyroid cancer screening in Korea, and lung cancer screening data from the ongoing projects in Taiwan and Japan. The Asian Oceanian Society of Radiology could serve as a platform for international collaboration, and educational exchange between member societies was also highlighted in the forum. Most Asian radiology societies are looking forward to an expansion in the scale of international collaboration and for the provision of additional exchange programs in the future.

Keywords: Radiology; International cooperation; Screening; Asia; Oceania

\section{INTRODUCTION TO THE BACKGROUND}

The Asian Radiology Forum (ARF) was first organized by the Korean Society of Radiology (KSR) to intensify intersociety collaboration in Asia. In March 2017, the third ARF was held with the annual congress meeting of the Chinese Taipei Society of Radiology (CTSR) in Taipei, focusing on "screening programs in Asia" and discussion on current landscape of screening and the evolving role of radiologists.

National delegates of the following radiological societies from Asia, including the president or executive members, were invited to this meeting to introduce their society

Received July 2, 2019; accepted after revision November 7, 2019. Corresponding author: Yeun-Chung Chang, MD, PhD, Department of Medical Imaging, National Taiwan University Hospital, No.7, Chung-Shan South Road, Taipei 100, Taiwan.

- Tel: (8862) 2356-2570 - Fax: (8862) 2322-4552

-E-mail: ycc5566@ntu.edu.tw

This is an 0pen Access article distributed under the terms of the Creative Commons Attribution Non-Commercial License (https://creativecommons.org/licenses/by-nc/4.0) which permits unrestricted non-commercial use, distribution, and reproduction in any medium, provided the original work is properly cited. and present their opinions on current imaging screening programs. Delegates from the CTSR, Indonesian Society of Radiology (ISR), Myanmar Radiological Society (MRS), Radiological Society of Thailand (RST), Indian Radiological and Imaging Association (IRIA), Hong Kong College of Radiologists (HKCR), Singapore Radiological Society (SRS), Japanese Radiological Society (JRS), and KSR presented their current status in this meeting.

The representatives demonstrated the status of the society and the role of radiologists in local screening programs. In this paper, we report the most important opinions presented by the participants of the ARF in 2017 and the discussions and conclusions about the current stands and future plans for intersociety collaboration in different radiological societies of Asia (Fig. 1).

\section{Current Status of the Society and Screening Programs}

\section{Indonesian Society of Radiology}

The ISR has 1200 members with approximately 250 active members and specifically 24 branches across Indonesia. The Indonesian College of Radiology (ICR) is a committee 


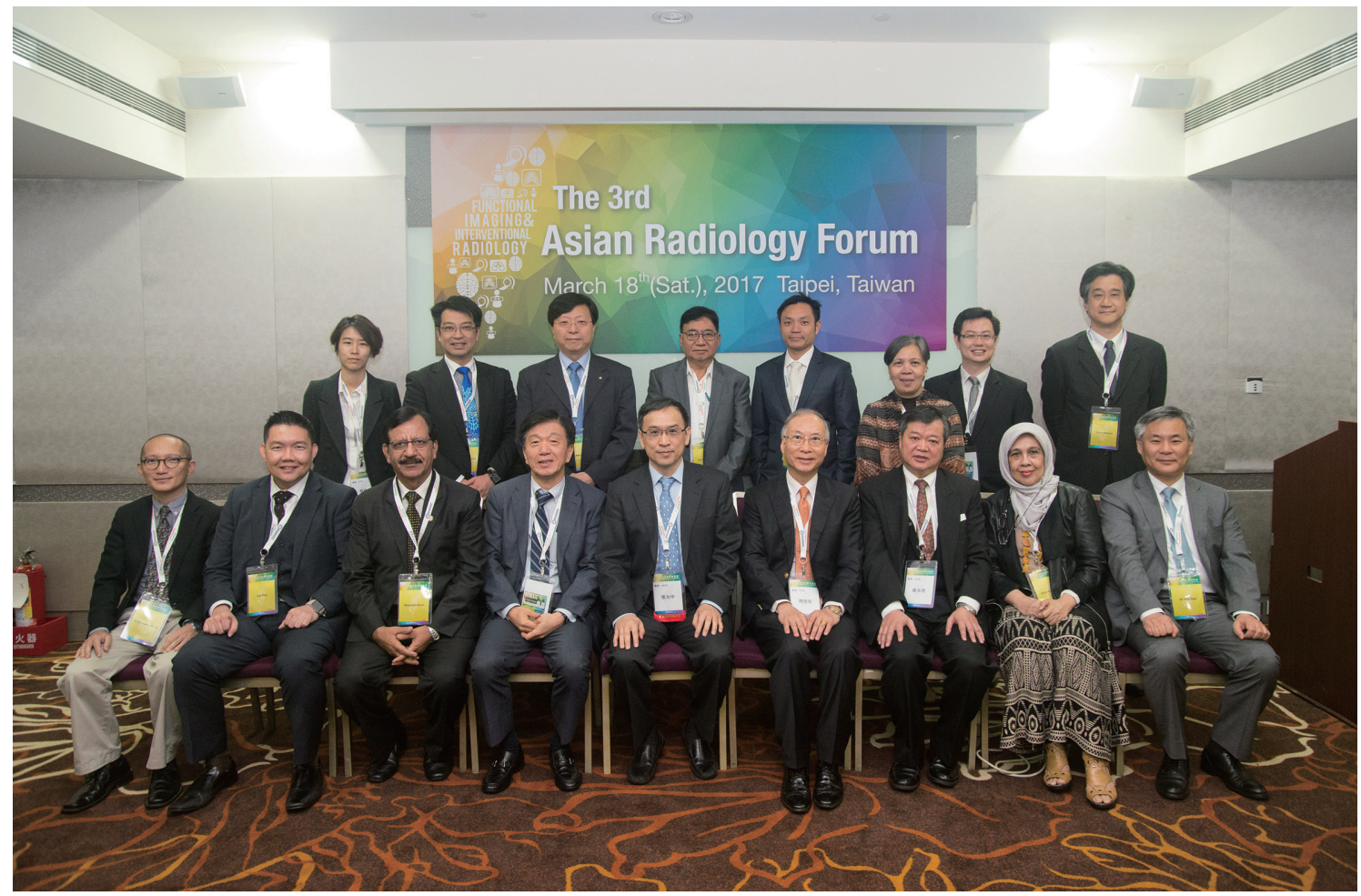

Fig. 1. Photograph of delegates from participating societies at Asian Radiology Forum 2017 in Taipei. From right to left in first row are Jin-Mo Goo from (Korea Society of Radiology), Aziza G. Icksan (President, Indonesian Society of Radiology), Yung-Liang Wan (CTSR), Yi-Hong Chou (President, AOSR), Yeun-Chung Chang (President, CTSR), Byung-Ihn Choi (Councilor, AOSR), Bhupendra Ahuja (President, Indian Radiological and Imaging Association), Pua Uei (Council member, AOSR), Chi-Wai Stephen Cheung (HKCR). On second row from right to left are Ning Chien (Deputy Secretory, CTSR), Danny Hing-Yan Cho (HKCR), Jong Min Lee (Councilor, AOSR), Tun Tun Lin (President, Myanmar Radiological Society), Cher-Heng Tan (Vice President, Singapore Radiological Society), Kardinah (Indonesia Society of Radiology), Surachate Siripongsakun (Royal College of Radiologists of Thailand), Kazuto Ashizawa (Japan Radiological Society). AOSR = Asian Oceanian Society of Radiology, CTSR $=$ Chinese Taipei Society of Radiology, HKCR = Hong Kong College of Radiologist

established by the ISR to support radiology education and set up a curriculum for radiology training and cancer screening.

Considering that population-based screening programs do not currently exist, there are guideline manuals issued by the Ministry of Health for multidisciplinary healthcare providers in referral hospitals. Moreover, the ISR and ICR are in the process of strengthening the subspecialty with all the related common cancers. The ISR and ICR are continuously educating general radiologists for improved accuracy in cancer diagnosis with imaging modalities through education. As a result, the ISR and ICR have promoted the establishment of the Indonesian Society of Breast and Women Reproductive Imaging, thus enhancing the role of radiologists in cancer screening for women. The president and delegate of the ISR, Professor Aziza G. Icksan, had stated that future collaboration with member societies for screening is anticipated, specifically on the prevalence and cost-effectiveness studies using the platform of the Asian 0ceanian Society of Radiology (AOSR). Furthermore, recommendation of a guideline for a high-risk lung cancer screening program endorsed by the AOSR would be significantly helpful to promote public screening policy in the society.

\section{Royal College of Radiologists of Thailand}

Founded in 1962, the Royal College of Radiologists of Thailand (RCRT) is a government organization under the Medical Council of Thailand and is composed of 8000 members, including 1500 working in diagnostic radiology, 200 in radiotherapy, and 100 in nuclear medicine. With a population of 70 million, the most common cancer among men in Thailand is liver cancer, followed by lung and colon cancers. However, in women, the most common cancers are breast, liver, and then cervical cancers. The liver cancer prevalence in Thailand is higher than that of other Asian countries, with an estimated national prevalence of up to $8.7 \%$ (more than six million patients) until 
2016. Moreover, in liver fluke-endemic areas in Northern Thailand, the demographics of liver cancer consist of mostly cholangiocarcinoma, resulting from chronic fluke infection attributed to consuming raw or undercooked fish. The national campaign project for cholangiocarcinoma offers abdominal ultrasound screening in these endemic areas and is now the first priority among other programs. The RCRT, RST, and Medical Ultrasonic Society of Thailand have collaborated and embarked on a training course for nurses in the targeted areas to strengthen the quality and accessibility of the sonography screening program. The RCRT looks forward to sharing and exchanging experiences on sonography screening programs.

\section{Myanmar Radiological Society}

The MRS was established in 1972 and currently has 350 members. The MRS is also a member of the AOSR. With a population of 53 million, there are 1056 public hospitals with 56746 beds in the country. In the absence of a population-based screening program, the estimated number of newly diagnosed cancer every year was 64000 according to the International Agency for Research on Cancer in 2012. The leading cancers among men are lung, stomach, and colorectal cancers. In women, breast, stomach, and cervical cancers are most common. There is sporadic screening for breast and cervical cancer with mammography and Pap smears in tertiary centers. Considering the rapid increase in cancer cases and the limited number of radiologists, the MRS needs international support, particularly with respect to diagnostic and treatment training programs, and requires the recruitment of additional radiologists for advanced technologies. The MRS seeks close collaboration with both national and international partners for improving cancer diagnosis, interventional procedure training, and also for advanced multidisciplinary team experiences.

\section{Hong Kong College of Radiologists}

The HKCR was incorporated in 1991 as one of the 15 constituent colleges of the Hong Kong Academy of Medicine. With a population of seven million, the HKCR has 335 qualified diagnostic radiologists. Currently, only regional cancer screening exists, including cervical cancer screening with Pap smears and the colon cancer screening program with stool fecal immunochemical test but it does not involve imaging diagnosis. Imaging is only performed when colonoscopy is not complete.

Breast cancer screening is mostly conducted in private imaging centers, with the Tung Wah Group of Hospitals is considered as the largest healthcare facility providing this service; until now, population screening programs do not exist. The number of screened cases has been increasing to up to more than 21000 annually since 1993 . Double reading and physical examination are routine protocols. The detection rate for early cancer is high $34.6 \%$ for ductal carcinoma in situ and $52.6 \%$ for minimal cancer; crude cancer detection rate, 5.2 per 1000). Overall, the screening program is targeted at women between 40 and 49 years old and supported by the Hong Kong Anti-Cancer Society because women younger than 45 years old accounted for $27 \%$ of all breast cancers in Hong Kong, according to the vice president of the HKCR, Chi Wai Stephen Cheung. In the current stance and intention for intersociety collaboration, the HKCR aims to further strengthen the support of young radiologists from neighboring countries and to closely collaborate with regional and specialty societies.

\section{Singapore Radiological Society}

In 2014, there were a total 26 hospitals with 11230 hospital beds in Singapore. The SRS consists of 337 registered radiologists and 114 residents.

Cancer is also the leading cause of death in Singapore with an incidence similar to that of other neighboring countries. The leading cancers among men and women are colorectal, lung, and prostate cancers, and breast, colorectal, and corpus uteri cancers, respectively.

An image-based breast cancer screening program was started in 2003, and both mammography and ultrasound are available in all centers. Moreover, annually, the National Breast Cancer Awareness Month is celebrated every month of October, and women greater than 50 years old are encouraged to join the screening program. The average screening rate is approximately $40 \%$.

Colorectal cancer is the most prevalent cancer in Singapore, while stool fits and colonoscopy remained the major screening tools. Computed tomography (CT) colonography and rectal magnetic resonance imaging (MRI) are elective but available in all facilities.

Low-dose CT (LDCT) lung cancer screening has not been widely adapted, and CT is still considered the standard imaging modality in cancer staging.

Assessment of prostate-specific antigen level is still considered the first step in prostate cancer screening, and MRI and prostate-specific membrane antigen positron emission tomography are increasingly used 
when clinically detecting recurrence and in targeted biopsy. Hepatocellular carcinoma screening programs offer ultrasound in combination with serum alpha fetoprotein level assessment for high-risk patients. The SRS had been closely collaborating with fellow societies, including the American Roentgen Ray Society (ARRS), American Institute for Radiologic Pathology (AIRP), Asia Pacific Society of Cardiovascular and Interventional Radiology (APSVIR), American Society of Neuroradiology (ASNR), and AOSR.

\section{Indian Radiological and Imaging Association}

Professor Bhupendra Ahuja is the president of the IRIA. The IRIA was established in 1931 and is a registered society with more than 12000 members. The IRIA specifically organizes programs to educate residents and young faculty members through its academic part "Indian College of Radiology and Imaging."

India has an estimated population of 1,048,000,000 with an estimated 9,500,000 newly diagnosed cancer cases and more than 700000 cancer deaths annually. It is also estimated that the incidence of all cancers would increase up to 1.7 million annually by 2035 . The most common cancers in India among men are oral and lung cancers, followed by stomach and colorectal cancers. In women, the most common ones are breast, cervical, and colorectal cancers. Over $75 \%$ of the patients report for treatment at advanced stages, resulting in poor survival rates. In November 2016, a human papillomavirus vaccination program was started in school-aged girls in Delhi.

Considering that a population-based screening program for cancer in India does not yet exist, there are still screening protocols in public facilities that are targeted at breast and ovarian cancers in symptomatic or high-risk patients. The associations and radiologists aim to improve the positive predictive values of transvaginal sonography and mammography.

IRIA representative, Bhupendra Ahuja, stated that intersociety collaboration should be encouraged through increasing collective academic symposiums among member societies. The IRIA aims to promote specific opportunities for collaboration, including joint annual meetings, research collaboration, and exchange of fellowships.

\section{Japanese Radiological Society}

The JRS was established in 1940 and currently has 9178 members, including 6149 board-certified radiologists. The Japan Radiology Congress, is considered the largest annual meeting in the JRS.

The incidence rate of cancer in Japan has been continuously increasing since the 1980s. Colorectal cancer had the highest incidence rate in 2012 for both sexes, followed by stomach, lung, and liver cancers. The mortality rate of cancer has been continuously increasing for both sexes since the 1960s. For both sexes, the mortality rate of lung, pancreas, and colorectal cancers increased, while the mortality rate of stomach and liver cancers decreased.

The incidence rates of stomach and liver cancers have been decreasing over time. In 2014, the cancer site with the highest mortality rate in Japan was the lung in both sexes, followed by the colon/rectum, stomach, pancreas, and liver. In 2011, it was estimated that more than 70000 deaths were reported due to lung cancer in Japan. A screening program for these five targeted cancers is recommended by the Japanese Ministry of Health, Labour and Welfare; they are stomach, colon, lung, breast, and uterine cancers, as stated by Professor Azuto Ashizawa.

According to the evidence of mortality reduction in chest radiograph screening studies conducted in Japan in the 1990s, the guidelines issued by the ministry suggest that heavy smokers should undergo annual chest X-ray and sputum cytology examinations. A population-based lowdose lung cancer screening trial was conducted in Hitachi City starting in 1995, recruiting a total of 31739 cases and 833342 tests as of March 2009. During the third period (2005-2009), which corresponds to 4-8 years after the introduction of $\mathrm{CT}$, a statistically significant $24 \%$ reduction in lung cancer mortality for men and women combined was observed in Hitachi City. Another ongoing population-based screening program has just been initiated in 2010, which was the Japanese randomized trial to evaluate the Efficacy of low-dose thoracic CT Screening (JECS) study. The study aimed to compare the screening efficacy between LDCT and chest radiography and enrolled a total of 27000 patients who were nonsmokers or had history of smoking less than 30 pack-years. The CT arm group underwent LDCT at 1 and 6 years, while the chest radiograph group underwent one chest radiography at baseline and 9 screenings based on the ministry's protocol. The follow-up interval was 10 years, and endpoint was lung cancer mortality.

In conclusion, LDCT is not recommended as a populationbased screening tool but accepted as an optional test for now. More evidences are anticipated from the ongoing studies. 


\section{Korean Society of Radiology}

The KSR was established in 1945 with the mission to promote the advancement of radiology and related sciences and medical services through collaboration among its members, to establish advisable healthcare policies and foresee their implementation for the promotion of national health, to protect its members' rights and interests, and to ensure a practice environment suitable for quality medical services. The KSR currently has 4368 members, including 3764 board-certified radiologists and 604 residents and 938 international memberships from India, Malaysia, Indonesia, Philippines, and Thailand. The KSR covers 12 affiliated subspecialty societies based on organ systems. Among the 4368 practicing radiologists, $57.5 \%$ are subspecialized radiologists, while $42.5 \%$ are general radiologists.

The National Cancer Screening Program in Korea includes five targeted cancers such as stomach, colorectal, liver, breast, and cervical cancers and also quality control programs for assessing health screening centers, as stated by delegate professor Jin Mo Goo. Screening involves imaging such as abdominal sonography for liver cancer, upper gastrointestinal (GI) barium study for stomach cancer, and mammography for breast cancer. The participation rates for the national cancer screening program have been increasing during the past decade. As of 2012, the participation rates ranged from $25 \%$ for colorectal screening up to $52 \%$ for breast cancer screening. Quality assessment of medical institutions for cancer screening started from 2008 with missions including upper GI barium studies and mammography education.

Regarding lung cancer screening, the Korean Lung Cancer Screening Project has just been initiated, recruiting 8000 participants from 11 centers focusing on adults aged 55 to 74 years old with smoking history greater than 30 packyears. The project incorporates network-based computer aided diagnosis and building a cloud-based database.

\section{Chinese Taipei Society of Radiology}

The Radiological Society of the Republic of China was founded in 1951 and renamed as the CTSR in 1991. It serves as a consultant to the government for medical care policy and has 1116 radiologist members and 184 trainees for a total of 23 million populations. There are 9 different subspecialty societies based on organ systems.

As many as $20 \%$ of the individuals who die from lung cancer in the USA annually are nonsmokers, and the estimated nonsmoker cancer percentage is even higher in Taiwan than that in other countries. As a result, the CTSR, Taiwan Lung Cancer Society, and Taiwan Society of Pulmonary and Critical Care Medicine conducted a prospective, nationwide multicenter trial dedicated for lung cancer screening for nonsmokers in 2015.

A total of 4498 subjects were enrolled with the following inclusion criteria: aged between 55 and 75 year old, had nonsmoking or light ex-smoking history (< 10 pack-years or have quit smoking $>15$ years), and had one of the following risk factors: family history of lung cancer $(n=1738,38.9 \%)$ and second-hand smoke $(n=3382,75.2 \%)$. Of the 4498 subjects, $4395(97.7 \%)$ met the inclusion criteria. A total of $19.9 \%$ of the 4395 subjects were considered positive on LDCT, and 1.64\% (72/4385) underwent invasive procedures for further treatment. The effective radiation dose was 1.064 $\pm 0.316 \mathrm{mSv}$.

The final pathology was as follows: 16 benign lesions $(0.4 \%)$, including 3 atypical adenomatous hyperplasia. A total of 56 patients $(1.3 \%)$ were diagnosed with lung cancer, including 6 cases of adenocarcinoma in situ, 10 cases of microinvasive adenocarcinoma, and 40 cases of invasive adenocarcinoma. Of these 56 patients, $96 \%$ had less than stage I cancer.

According to the International Early Lung Cancer Action Program, a total of 57496 baseline screenings were performed, and $4.2 \%$ (2392) of the patients had groundglass nodules (GGNs), among which 26\% (628) resolved or regressed spontaneously. Lung cancer was diagnosed in 73 patients ( $3 \%$ of GGN; $0.13 \%, 1.3$ cases per 1000 baseline screenings).

However, in the current preliminary study regarding GGNs, $27.5 \%(8 / 29)$ of patients with GGN were diagnosed with adenocarcinoma, and 20\% (8/40) of all adenocarcinomas presented as pure GGN. Our data proved that GGNs in Taiwan have different demographics and behaviors. However, considering the limited number of positive cases (56 cancers out of the 4395 cases), quantifying risk factors such as second-hand smoke is relatively difficult, and the solid component of the part-solid nodule was not analyzed in the study. The prevalence of lung cancer in highrisk nonsmokers from LDCT screening was $1.3 \%$, which is comparable to that of $1.1 \%$ in the high-risk smoking group in the National Lung Screening Trial.

\section{CONCLUSION}

The forum, which was focused on screening the landscape 
across Asia demonstrated various cancer demographics and screening protocols. In addition to increasing awareness and the importance of radiologists in screening programs, the need for educational programs and exchange on trial experiences were also highlighted in the forum. Most Asian radiology societies look forward to expanding partner societies and enhancing globalization and collaboration programs through the AOSR platform. 